Commun. Math. Phys. 139, 71-82 (1991)

Communications in

Mathematical

Physics

(C) Springer-Verlag 1991

\title{
Conformal Field Theories via Hamiltonian Reduction
}

\author{
Michael Bershadsky* \\ The Institute for Advanced Study, Princeton, New Jersey 08540, USA
}

Received May 30, 1990

\begin{abstract}
Constraining the $S L(3)$ WZW-model we construct a reduced theory which is invariant with respect to the new chiral algebra $W_{3}^{2}$. This symmetry is generated by the stress-energy tensor, two bosonic currents with spins $3 / 2$ and the $U(1)$ current. We conjecture a Kac formula that describes the highly reducible representation for this algebra. We also discuss the quantum Hamiltonian reduction for the general type of constraints that leads to the new extended conformal algebras.
\end{abstract}

\section{Introduction}

It was recently observed $[1,2]$ there are hidden relations between the Virasoro algebra and the $S L(2, \mathscr{R})$ current algebra, and in general, between the $W_{n}$-algebra and the $S L(n, \mathscr{R})$ current algebra. The $W_{n}$-algebra is an extension of the Virasoro algebra with additional chiral operators of spin $n[3,4]$.

The relation between the current algebras and the extended conformal algebras is given by Drinfeld-Sokolov [5] reduction. One may regard the space dual to the loop algebra as the phase space endowed with a natural symplectic structure. This phase space possesses a certain symmetry and one may consider the reduced phase space under this symmetry. The Poisson Brackets on the reduced phase space are given by Drinfeld-Sokolov reduction [5] and coincide with the classical limit of extended conformal algebras.

Quantizing such theory one must replace the Poisson Brackets by commutation relations and classical phase space by an irreducible representation of the algebra. The irreducible representation spaces of the Virasoro algebra are extracted from those of the $S L(2, \mathscr{R})$ current algebra by imposing a certain constraint on the latter. Consider an irreducible representation space of the current algebra. In classical mechanics, we put a constraint $J^{-}(z)=1$ to reduce the phase space of the loop algebra. Quantum mechanically, we introduce a set of ghosts and define the

* Address after September 1990: Lyman Laboratory, Harvard University, Cambridge, MA 02138, USA 
Becchi-Rouet-Stora-Tyutin (BRST) operator associated with this constraint. An irreducible representation space of the Virasoro algebra is isomorphic to a quotient $\operatorname{Ker}\left(Q_{\text {BRST }}\right) / \operatorname{Im}\left(Q_{\text {BRST }}\right)$.

This construction may be used in order to construct the chiral algebras. For example, starting from $S L(n, \mathscr{R})$ current algebra and constraining the currents from the Borel subalgebra one ends up with the $W_{n}$ algebra [2]. In this paper we discuss the following general type of constraints. Define the weight $\delta=\sum_{j} \omega_{j}$, where $\omega_{i}$ are the fundamental weights for $S L(n, \mathscr{R})$. For any integer $1 \leqq l \leqq(n-1)$ and for all positive roots $e$ define the constraints as follows:

$$
J_{-e}(z)= \begin{cases}1, & \text { if }\langle\delta e\rangle=l \\ 0, & \text { otherwise }\end{cases}
$$

Let us call these constraints of degree $l$. We constrain the currents from the $l^{\text {th }}$ upper diagonal to be equal to one. The rest of the currents from the Borel subalgebra are equal to zero. For $l=1$ we recover the standard constraints that yield the well known $W_{n}$ algebras. For $l>1$ some constraints are of the second class so one must be careful quantizing such systems. We claim that in general these constraints lead to new extended conformal algebras $W_{n}^{l}$. There is a lot of evidence that these algebras possess highly reducible representations that correspond to minimal models. We present here the discrete values of the conformal anomaly for the $W_{n}^{2}$ models:

$$
c=1-\frac{1}{2}\left(n^{3}-n\right) \frac{(p-q)^{2}}{p q} .
$$

For simplicity we consider the $S L(3, \mathscr{R})$ WZW theory. There are two possibilities of constraining this model

$$
J=\left(\begin{array}{lll}
* & 1 & 0 \\
* & * & 1 \\
* & * & *
\end{array}\right) \quad J=\left(\begin{array}{lll}
* & 0 & 1 \\
* & * & 0 \\
* & * & *
\end{array}\right) .
$$

The first possibility gives the $W_{3}$ algebra, while the second leads to the new chiral algebra $W_{3}^{2}$. Both these theories possess a hidden $S L(3, \mathscr{R})$ symmetry which is gauged and hidden, but it is gauged differently. It would be very interesting to understand if there is direct relation between $W_{3}$ and $W_{3}^{2}$. The generalization for the $S L(n, \mathscr{R})$ WZW model is straightforward.

In understanding various aspects of conformal field theories, it has proved fruitful to explore the interplay between the Virasoro algebra and the complex geometry of Riemann surfaces. Is there also some geometrical structure behind these algebras? To answer this question, we must understand what kind of symmetry these algebras imply. The Virasoro algebra is the consequence of reparameterization and Weyl scaling invariance of a field theory, and the structure of these symmetries is encoded into the geometric action of the Virasoro algebra. Thus the first step would be to construct a geometric action for these algebras. Quantum Hamiltonian reduction makes it possible to define a natural generalization for the geometric action.

The paper is organized as follows. In Sect. 2 we study the geometric action for the algebras in question, discuss its symmetries and construct the nilpotent $Q_{\text {BRST }}$ operator. In Sect. 3 we consider the simplest example: $S L(3, \mathscr{R})$ constrained by the 
constraints of degree 2. The nilpotent BRST operator acts on the total space which is the product of the representation space of $S L(3, \mathscr{R})$, the Fock space of the auxiliary fields and the appropriate ghost Fock space. We reduce the $S L(3, \mathscr{R})$ screening operators by the $Q_{\text {BRST }}$ that impose the constraints. Then we work out the explicit structure of the chiral algebra as the commutant of reduced screening operators and discuss the bosonization rules. The original suggestion by Polyakov [6] that this algebra may be related to $N=2$ superconformal algebra by statistic transmutation turns out to be wrong. Nevertheless these two algebras have many similarities. In the last section we discuss some generalizations for the $W_{n}^{2}$ algebra.

There is another interesting issue. The reduction of degree one (standard) corresponds to the $\mathrm{KdV}$ hierarchy, which is very popular today in the context of two-dimensional gravity $[7,8]$. We suspect that each type of reduction has its own hierarchy of nonlinear differential equations which may be relevant to twodimensional physics. We hope to return to this question in one of our future publications.

\section{Geometric Action}

In this section we consider the Constrained Wess-Zumino-Witten model and construct the geometric action. For simplicity we consider the $W_{3}^{2}$ case. The generalization for $W_{n}^{2}$ (or for $W_{n}^{l}$ ) is straightforward.

Before starting our discussion let us introduce some notations. The $\operatorname{SL}(3, \mathscr{R})$ current algebra is generated by six charged currents $J_{1}^{ \pm}, J_{2}^{ \pm}, J_{3}^{ \pm}$and two neutral currents $H_{1}, H_{2}$. The basis is chosen in such a way that these currents correspond to $S L(3)$ generators as

$$
\begin{aligned}
& J_{1}^{+} \leftrightarrow\left(\begin{array}{lll}
0 & 1 & 0 \\
0 & 0 & 0 \\
0 & 0 & 0
\end{array}\right) J_{2}^{+} \leftrightarrow\left(\begin{array}{lll}
0 & 0 & 0 \\
0 & 0 & 1 \\
0 & 0 & 0
\end{array}\right) J_{3}^{+} \leftrightarrow\left(\begin{array}{lll}
0 & 0 & 1 \\
0 & 0 & 0 \\
0 & 0 & 0
\end{array}\right) \\
& H_{1} \leftrightarrow\left(\begin{array}{ccc}
1 & 0 & 0 \\
0 & -1 & 0 \\
0 & 0 & -0
\end{array}\right), \quad H_{2} \leftrightarrow\left(\begin{array}{ccc}
0 & 0 & 0 \\
0 & 1 & 0 \\
0 & 0 & -1
\end{array}\right) .
\end{aligned}
$$

Classically one has to impose the constraints

$$
J_{1}^{-}(z)=J_{2}^{-}(z)=0, \quad J_{3}^{-}(z)=1 .
$$

In order to make these constraints consistent with conformal invariance one has to modify the Sugawara stress-energy tensor for the $S L(3, \mathscr{R})$ current algebra in such a way that the current $J_{3}^{-}$acquire zero dimension

$$
T_{\text {improved }}(z)=T_{S L(3)}-\frac{1}{2} \partial\left(H_{1}(z)+H_{2}(z)\right) \text {. }
$$

With respect to this stress-energy tensor the fields $J_{1,2}^{-}$have weights $1 / 2$. The constraints (5) are of second class. In the previous paper [9] we gained some experience how to treat second class constraints. To obtain first class constraints let us introduce a pair of conjugate auxiliary fields $\chi(z)$ and $\chi^{+}(z)$

$$
\chi(z) \chi^{+}(w) \sim \frac{1}{(z-w)},
$$


and consider the quantum constraints

$$
J_{1}^{-}(z)=\chi(z), \quad J_{2}^{-}(z)=\chi^{+}(z), \quad J_{3}^{-}(z)=1 .
$$

The total stress-energy tensor is the sum of three pieces: the modified stress-energy tensor (6), the stress-energy tensor of the auxiliary system and the ghost stressenergy tesnor. The ghost fields naturally emerge if we impose the constraints (8) using the BRST formalism. The central charge for this total stress-energy tensor is

$$
c_{k}=\frac{8 k}{k+3}-6 k-1=25-\frac{24}{k+3}-6(k+3) .
$$

The third term -1 is the contribution of the auxiliary fields $\chi, \chi^{+}$. The ghost conformal anomaly turns out to be zero. It is convenient to parameterize the level $k+3=2 p / q$. Then the formula for the conformal anomaly becomes

$$
c=1-12 \frac{(p-q)^{2}}{p q} \text {. }
$$

Now we are ready to formulate the constrained WZW-model coupled to the auxiliary fields $\chi, \chi^{+}$,

$$
\begin{aligned}
S_{\text {gauged }}\left(g, \bar{A}^{+}, \chi, \chi^{+}\right)= & k S_{\text {wzw }}(g)-\int \frac{d^{2} z}{8 \pi} \chi \bar{\partial} \chi^{+} \\
& +\int \frac{d^{2} z}{8 \pi}\left[\bar{A}_{3}^{+}\left(J_{3}^{-}-1\right)+\bar{A}_{1}^{+}\left(J_{1}^{-}-\chi\right)+\bar{A}_{2}^{+}\left(J_{2}^{-}-\chi^{+}\right)\right] .
\end{aligned}
$$

The fields $\bar{A}_{i}^{+}$are the Lagrange multipliers that impose constraints. The gauged WZW action $S_{\text {gauged }}\left(g, \bar{A}^{+}, \ldots\right)$ possesses a gauge symmetry. Under the multiplication

$$
g \rightarrow U g, \quad U=\left(\begin{array}{ccc}
1 & 0 & 0 \\
u_{1} & 1 & 0 \\
u_{3} & u_{2} & 1
\end{array}\right)
$$

the field $\bar{A}^{+}$transforms as a gauge field

$$
\bar{A}^{+} \rightarrow U^{-1} \bar{A}^{+} U-U^{-1}(\bar{\partial} U), \quad \delta \chi=-u_{2}, \quad \delta \chi^{+}=u_{1} .
$$

The action (11) is also invariant with respect to residual symmetry $g \rightarrow g V(\bar{z})$.

Now let us count the degrees of freedom. The initial theory possesses $8+2$ degrees of freedom. The action $S_{\text {gauging }}\left(g, \bar{A}^{+}, \ldots\right)$ is invariant under a 3-parameter gauge group and there are also 3 constraints. Therefore the resulting theory possesses 4 degrees of freedom.

Quantizing this theory via the BRST formalism one needs to introduce the ghost fields: a $(b, c)$ system of dimension $(0,1)$ and two sets of $\left(\xi_{i}, \eta_{i}\right)$ systems of dimension $\left(\frac{1}{2}, \frac{1}{2}\right)$. By adding the appropriate BRST exact operator to the action one imposes the gauge fixing conditions $\bar{A}_{i}^{+}=0$. Therefore we obtain the relation

$$
\begin{aligned}
& \int \frac{\left[g^{-1} d g, d \bar{A}^{+}, d \chi, d \chi^{+}\right]}{\text {(gauge volume) }} \exp \left(i S_{\text {gauged }}\left(g, \bar{A}^{+}, \chi, \chi^{+}\right)\right) \\
& \quad=\int\left[g^{-1} d g, \ldots\right] \exp \left(i k S_{\mathrm{wzw}}(g)+i S_{\text {ghost }}\left(c, b, \xi_{i}, \eta_{i}\right)-i \int \frac{d^{2} z}{8 \pi} \chi \bar{\partial} \chi^{+}\right) .
\end{aligned}
$$


The BRST transforms of $g$ and $\chi, \chi^{+}$are defined by replacing the parameters $u_{1}, u_{2}, u_{3}$ of the infinitesimal gauge transformation by the ghost fields $\eta_{1}, \eta_{2}, c$ (see (12)-(13)). The on-shell BRST transformations of the ghost-antighost fields are

$$
\begin{aligned}
\delta_{\mathrm{BRST}} \xi_{1} & =0, \quad \delta_{\mathrm{BRST}} \xi_{2}=0, \quad \delta_{\mathrm{BRST}} c=\xi_{1} \xi_{2}, \\
\delta_{\mathrm{BRST}} b & =J_{3}^{-}-1, \quad \delta_{\mathrm{BRST}} \eta_{1}=J_{1}^{-}-\chi-b \xi_{2}, \quad \delta_{\mathrm{BRST}} \eta_{2}=J_{2}^{-}-\chi^{+}+b \xi_{1} .
\end{aligned}
$$

These transformations are generated by the nilpotent BRST operator

$$
Q_{\mathrm{BRST}}=\int \frac{d z}{2 \pi}\left[c\left(J_{3}^{-}-1\right)+\xi_{1}\left(J_{1}^{-}-\chi\right)+\xi_{2}\left(J_{2}^{-}-\chi^{+}\right)+b \xi_{1} \xi_{2}\right]
$$

We postpone the discussion of the properties of $Q_{\mathrm{BRST}}$ to the next section.

To construct the geometric action we follow the procedure developed by Alekseev and Shatashvili [10]. Starting from the path integral (14) one can first integrate over $\bar{A}^{+}$,

$$
\int \frac{\left[g^{-1} d g, d \chi, d \chi^{+}\right]}{(\text {gauge volume })} \delta\left(J_{3}^{-}(z)-1\right) \delta\left(J_{1}^{-}(z)-\chi\right) \delta\left(J_{2}^{-}(z)-\chi^{+}\right) \exp \left(i S\left(g, \chi, \chi^{+}\right)\right) .
$$

It is easy to show that the above path integral still has the Borel gauge invariance $g \rightarrow U g, \delta \chi=-u_{2}, \delta \chi^{+}=u_{1}$ that enable us to impose the gauge conditions $\delta \chi=\delta \chi^{+}=0, H_{3}=0$. Using the Gauß decomposition

$$
g=\left(\begin{array}{ccc}
1 & 0 & 0 \\
\phi_{1} & 1 & 0 \\
\phi_{3} & \phi_{2} & 1
\end{array}\right) \cdot\left(\begin{array}{ccc}
\lambda & 0 & 0 \\
0 & \mu & 0 \\
0 & 0 & \lambda^{-1} \mu^{-1}
\end{array}\right) \cdot\left(\begin{array}{ccc}
1 & F_{1} & F_{3} \\
0 & 1 & F_{2} \\
0 & 0 & 1
\end{array}\right),
$$

we may rewrite the constraints (8) and the gauge condition $H_{3}=0$ as follows:

$$
\begin{aligned}
& J_{1}^{-}=\lambda \mu^{-1} \partial F_{1}-\phi_{2}=0, \quad J_{2}^{-}=\lambda \mu^{2} \partial F_{2}+\phi_{1}=0, \\
& J_{3}^{-}=\lambda^{2} \mu\left(\partial F_{3}-F_{2} \partial F_{1}\right)=1, \\
& H_{3}=2 \lambda^{-1} \partial \lambda+\mu^{-1} \partial \mu+\phi_{1} \phi_{2}-2 \phi_{3}=0 .
\end{aligned}
$$

By solving them with respect to $\lambda, \mu, F_{2}$ and $\phi_{3}$ and substituting them into the WZW action, we obtain the effective theory for $F_{1}, F_{3}, \phi_{1}$ and $\phi_{2}$. The geometric action is

$$
S_{\text {geometric }}\left(F_{1}, F_{3}, \phi_{1}, \phi_{2}\right)=S_{\mathrm{WZw}}(g)
$$

evaluated under the constraints (19). The dynamical variables we choose do not require any additional Jacobian factor in the path integral. The choice of dynamical variables is not unique. One may for example choose $\lambda, \mu, F_{1}, F_{3}$ as the dynamical variables. The measure of integration is the reduced Haar measure for the $S L(3, \mathscr{R})$. The effective theory (20) has the left moving $S L(3, \mathscr{R})$ current algebra while the right sector is invariant under the $W_{3}^{2}$ algebra. The origin of this symmetry is the following. The constrained WZW model is no longer invariant with respect to the current algebra. An infinitesimal gauge transformation spoils the constraints. But one may project back into the gauge slice. The combination of gauge transformation and the projection will be precisely the symmetry of the reduced theory. The chiral algebra $W_{3}^{2}$ is generated by two bosonic fields $G^{-}(z)$ and $G^{+}(z)$ of weights $3 / 2$, the stress-energy tensor $T(z)$ and the $U(1)$ current $H(z)$. Roughly speaking, $G^{+}(z)=J_{1}^{+}(z)$, 
$G^{-}(z)=J_{2}^{+}(z)$ and the stress-energy tensor $T(z)$ is a linear combination of $J_{3}^{+}(z)$ and $H^{2}(z)$. One can easily deduce the classical bosonization rules in terms of free fields $\vec{\varphi}(z), \phi(z)=\phi_{2}(z)$ and $\phi^{+}=\phi_{1}(z)$,

$$
\begin{aligned}
H(z)= & \frac{\sqrt{2}}{3}\left(\vec{e}_{2}-\vec{e}_{1}\right) \partial \vec{\varphi}+\varphi(z) \phi^{+}(z), \\
G^{+}(z)= & \partial \phi^{+}+\sqrt{2}\left(\vec{e}_{1} \partial \vec{\varphi}\right) \phi^{+}(z)-\left(\phi^{+}(z)\right)^{2} \phi(z), \\
G^{-}(z)= & \partial \phi+\sqrt{2}\left(\vec{e}_{2} \partial \vec{\varphi}\right) \phi(z)+\phi^{+}(z) \phi^{2}(z), \\
T(z)= & \frac{2}{3}\left[\left(\vec{e}_{1} \partial \vec{\varphi}\right)^{2}+\left(\vec{e}_{2} \partial \vec{\varphi}\right)^{2}+\left(\vec{e}_{1} \partial \vec{\varphi}\right)\left(\vec{e}_{2} \partial \vec{\varphi}\right)\right] \\
& +\frac{1}{\sqrt{2}}\left(\vec{e}_{1}+\vec{e}_{2}\right) \partial^{2} \vec{\varphi}+\frac{1}{2}\left(\partial \phi^{+} \phi-\partial \phi \phi^{+}\right),
\end{aligned}
$$

where $\vec{e}_{1}, \vec{e}_{2}$ are simple positive roots of $S L(3, \mathscr{R})$. The two-dimensional scalar field $\vec{\varphi}(z)$ bosonizes the Cartan subalgebra

$$
\lambda(z)=\exp \left(\frac{\sqrt{2}}{3}\left(2 \vec{e}_{1}+\vec{e}_{2}\right) \cdot \vec{\varphi}(z)\right), \quad \mu(z)=\exp \left(\frac{\sqrt{2}}{3}\left(\vec{e}_{2}-\vec{e}_{1}\right) \cdot \vec{\varphi}(z)\right) .
$$

The existence of this chiral algebra was originally suggested by Polyakov [6]. In the next section we construct the quantum bosonization rules.

\section{Operator Algebra}

To quantize the system with the constraints one may either quantize the system and impose the constraints afterwords or enforce the constraints first and then quantize the reduced system. Provided there is no anomaly these two procedures lead to the same quantum theory. In this section we employ the first possibility. We consider the Hilbert space of the $S L(3, \mathscr{R})$ WZW-model and impose the constraints via the BRST formalism. The total Hilbert space before imposing the constraints is the product of the representation space of the $S L(3, \mathscr{R})$ current algebra level $k$, auxiliary Fock space and the ghost Fock space,

$$
\mathscr{H}_{\text {total }}=\mathscr{H}_{S L(3)} \otimes \mathscr{H}_{\chi, \chi^{+}} \otimes \mathscr{H}_{\text {ghost }} .
$$

Let us start with the free boson realization of $S L(3, \mathscr{R})$ current algebra level $k$ in terms of two scalar fields $\vec{\varphi}=\left(\varphi_{1}, \varphi_{2}\right)$ and three sets of bosonic ghosts, $\left(\beta_{1}, \gamma_{1}\right)$, $\left(\beta_{2}, \gamma_{2}\right)$ and $\left(\beta_{3}, \gamma_{3}\right)$, with weights $(0,1),(0,1)$ and $(-1,2)$ respectively,

$$
\varphi_{a}(z) \varphi_{b}(w) \sim \frac{1}{2} \delta_{a b} \log (z-w), \quad \beta_{i}(z) \gamma_{j}(w) \sim \frac{\delta_{i j}}{z-w} \quad(a, b=1,2, i, j=1,2,3) .
$$

This realization may be found in ref. 2. Here we only remind the reader of the bosonization rules for the currents from the Borel subalgebra,

$$
J_{1}^{-}=\beta_{1}+\gamma_{2} \beta_{3}, \quad J_{2}^{-}=\beta_{2}, \quad J_{3}^{-}=\beta_{3}, \ldots .
$$

There are also four screening operators for the $S L(3, \mathscr{R})$ current algebra that play 
an important role in the representation theory,

$$
\begin{aligned}
& \Psi_{S L(3)}^{(1, \pm)}=\left(\beta_{1}(z)\right)^{n_{ \pm}} \exp \left(\alpha_{ \pm} \vec{e}_{1} \cdot \vec{\varphi}(z)\right), \\
& \Psi_{S L(3)}^{(2, \pm)}=\left(\beta_{2}(z)+\gamma_{1}(z) \beta_{3}(z)\right)^{n_{ \pm}} \exp \left(\alpha_{ \pm} \vec{e}_{1} \cdot \vec{\varphi}(z)\right),
\end{aligned}
$$

with

$$
n_{+}=-(k+3), \quad n_{-}=1 \text {, }
$$

where $k$ is the level of the current algebra. In spite of the fact that two of these screening operators $\Psi_{S L(3)}^{(1,+)}$ and $\Psi_{S L(3)}^{(2,+)}$ are ill defined, we would like to think of them as operators that can be defined meaningfully. Actually we need them to guess the form of the reduced screening operators. It is plausible that the construction of irreducible representations for $S L(2, \mathscr{R})$ using the screening operators [11] extends to the case of $S L(3, \mathscr{R})$. The total Hilbert space can be decomposed into the subspaces with definite charges with respect to $\partial \vec{\varphi}$. Using the screening operators $\Psi_{S L(3)}^{(i, \pm)}(i=1,2)$ one can define a complex which has a rather tricky structure and whose cohomology should yield the irreducible representation of $S L(3, \mathscr{R})$. We are not going to go deep into details and refer the reader to the original papers [12-15]. Denoting this cohomology as $H_{S L(3)}$ one may write

$$
\mathscr{H}_{\text {total }}=H_{S L(3)}\left(\otimes_{i} \mathscr{H}_{\beta_{i}, \gamma_{i}} \otimes \mathscr{H}_{\vec{\varphi}} \otimes \mathscr{H}_{\chi, \chi^{+}} \otimes \mathscr{H}_{\text {ghost }}\right) .
$$

The nilpotent BRST operator (16) acts on the total Hilbert space $\mathscr{H}_{\text {total }}$. Therefore one can immediately define the reduced Hilbert space of the constrained system as the cohomology space

$$
\mathscr{H}_{\text {red }}=\mathscr{H}_{Q_{\text {BRST }}}\left(\mathscr{H}_{\text {total }}\right)=\operatorname{Ker}\left(Q_{\text {BRST }}\right) / \operatorname{Im}\left(Q_{\text {BRST }}\right) \text {. }
$$

To describe the reduced Hilbert space let us represent the BRST operator in a form $Q_{\mathrm{BRST}}=Q_{0}+Q_{1}$, where

$$
\begin{aligned}
& Q_{0}=\int \frac{d z}{2 \pi}\left[c\left(\beta_{3}-1\right)+\xi_{1}\left(\beta_{1}+\gamma_{2}-\chi\right)+\xi_{2}\left(\beta_{2}-\chi^{+}\right)\right] \\
& Q_{1}=\int \frac{d z}{2 \pi}\left[b \xi_{1} \xi_{2}+\xi_{1} \gamma_{2}\left(\beta_{3}-1\right)\right] .
\end{aligned}
$$

Both $Q_{0}$ and $Q_{1}$ are nilpotent and anticommute. The crucial point is that $Q_{1}$ is $Q_{0}$ exact, namely $Q_{1}=\left[Q_{0}, R\right]$, where $R$ is also nilpotent. That implies the two nilpotent operators $Q_{\mathrm{BRST}}$ and $Q_{0}$ are related to each other by conjugation,

$$
Q_{\mathrm{BRST}}=e^{-R} Q_{0} e^{R}, \quad R=\int \frac{d z}{2 \pi} b \xi_{1} \gamma_{2} .
$$

The next step is to relate the reduced Hilbert space $\mathscr{H}_{\text {red }}$ to the cohomology space $\mathscr{H}_{S L(3)}\left(H_{Q_{\text {BRST }}}\right)$. To do that one needs to interchange the order of the cohomologies. For the case of $S L(2, \mathscr{R})$ the sufficient condition for this was formulated in ref. 2. Making the reasonable assumption that this condition is also satisfied for the $S L(3, \mathscr{R})$ case one can interchange the order of the cohomologies,

$$
H_{\text {red }}=H_{S L(3)}\left(H_{\text {BRST }}\left(\otimes_{i} \mathscr{H}_{\beta_{i}, \gamma_{t}} \otimes \mathscr{H}_{\vec{\varphi}} \otimes \mathscr{H}_{\chi, \chi^{+}} \otimes \mathscr{H}_{\text {ghost }}\right)\right) \text {. }
$$

Now let us relate the $Q_{\mathrm{BRST}}$ cohomologies to the $Q_{0}$ cohomologies. But $Q_{\mathrm{BRST}}$ and 
$Q_{0}$ differ by conjugation (see (31)) and therefore their cohomologies are isomorphic. Therefore one can simply replace the $Q_{\mathrm{BRST}}$ cohomologies by $Q_{0}$ cohomologies. The latter can be easily computed. Making a linear transformation of the field variables one finds that $\left(\beta_{1}+\gamma_{2}-\chi,-\chi^{+}\right)$and $\left(\xi_{1}, \eta_{1}\right),\left(\beta_{2}-\chi^{+}, \gamma_{2}+\beta_{1}\right)$ and $\left(\xi_{2}, \eta_{2}\right)$ and $\left(\beta_{3}, \gamma_{3}\right)$ and $(b, c)$ compose the Kugo Ojima [17] quartets and simply decouple from the physical subspace. Therefore the $Q_{\text {BRST }}$ cohomologies are isomorphic to

$$
H_{Q_{0}}=\left(\otimes_{i} \mathscr{H}_{\beta_{1}, \gamma_{i}} \otimes \mathscr{H}_{\bar{\varphi}} \otimes \mathscr{H}_{\chi, \chi^{+}} \otimes \mathscr{H}_{\text {ghost }}\right)=\mathscr{H}_{\vec{\phi}} \otimes \mathscr{H}_{\phi \phi^{+}},
$$

where $\left(\phi, \phi^{+}\right)=\left(\beta_{1}, \gamma_{1}+\beta_{2}\right)$.

Now let us define the reduced screening operators,

$$
\begin{aligned}
& V_{\text {red }}^{(1, \pm)}=(\phi(z))^{n_{ \pm}} \exp \left(\alpha_{ \pm} \vec{e}_{1} \cdot \vec{\varphi}(z)\right), \\
& V_{\text {red }}^{(2, \pm)}=\left(\phi^{+}(z)\right)^{n_{ \pm}} \exp \left(\alpha_{ \pm} \vec{e}_{2} \cdot \vec{\varphi}(z)\right) .
\end{aligned}
$$

The $S L(3, \mathscr{R})$ screening operators are BRST equivalent to the reduced screening operators up to conjugation

$$
e^{R} \Psi_{S L(3)} e^{-R}-V_{\text {red }}=\left[Q_{0}, *\right] .
$$

This is sufficient to demonstrate that

$$
\mathscr{H}_{\text {red }}=H_{\text {red }}\left(\mathscr{H}_{\vec{\varphi}} \otimes \mathscr{H}_{\phi, \phi^{+}}\right) \text {. }
$$

We denote by $H_{\text {red }}$ the cohomologies defined "a la Felder" $[16,11]$ for the reduced screening operators. As one should expect from classical considerations one can realize the physical space of the reduced system in terms of two scalar fields $\vec{\varphi}=\left(\varphi_{1}, \varphi_{2}\right)$ and two bosonic fields $\left(\phi, \phi^{+}\right)$with weights $(1 / 2,1 / 2)$. Now we come to the main issue. What is the symmetry of the reduced system and how can one construct it? There is a very nice description of the chiral algebras as the commutant of the screening operators. Therefore we just need to construct operators that commute with screening operators. This procedure leads directly to the bosonization rules and therefore the algebra constructed in such a way will be automatically associative.

It is easy to construct the $U(1)$-current and two fields $G^{+}(z)$ and $G^{-}(z)$ with weights $3 / 2$ and $U(1)$ charge \pm 1 ,

$$
\begin{aligned}
H(z) & =\frac{1}{3} \alpha_{+}\left(\vec{e}_{2}-\vec{e}_{1}\right) \partial \vec{\varphi}+\phi(z) \phi^{+}(z), \\
G^{+}(z) & =(k+1) \partial \phi^{+}+\alpha_{+}\left(\vec{e}_{1} \partial \vec{\varphi}\right) \phi^{+}(z)-\left(\phi^{+}(z)\right)^{2} \phi(z), \\
G^{-}(z) & =(k+1) \partial \phi+\alpha_{+}\left(\vec{e}_{2} \partial \vec{\varphi}\right) \phi(z)+\phi^{+}(z) \phi^{2}(z),
\end{aligned}
$$

where $\alpha_{+}=\sqrt{2 k+6}$. Commuting $G^{+}(z)$ and $G^{-}(z)$ one gets $H(z), H^{2}(z)$ and the stress-energy tensor $T(z)$,

$$
\begin{aligned}
T(z)= & \frac{2}{3}\left[\left(\vec{e}_{1} \partial \vec{\varphi}\right)^{2}+\left(\vec{e}_{2} \partial \vec{\varphi}\right)^{2}+\left(\vec{e}_{1} \partial \vec{\varphi}\right)\left(\vec{e}_{2} \partial \vec{\varphi}\right)\right] \\
& +\frac{(k+1)}{\alpha_{+}}\left(\vec{e}_{1}+\vec{e}_{2}\right) \partial^{2} \vec{\varphi}+\frac{1}{2}\left(\partial \phi^{+} \phi-\partial \phi \phi^{+}\right) .
\end{aligned}
$$

Let us also remind the reader of the normalization of the roots $e_{i}^{2}=2$. The 
constructed fields do not form a closed OPE algebra, but an algebra with quadratic relations. The central charge is given by the expression (9)

$$
c_{k}=25-\frac{24}{k+3}-6(k+3)
$$

The $U(1)$-current $H(z)$ is a conformal field with dimension 1 and satisfies the OPE,

$$
H(z) H(w) \sim \frac{(2 k+3) / 3}{(z-w)^{2}} .
$$

The fields $G^{ \pm}(z)$ are conformal fields with dimension $3 / 2$ and therefore satisfy the OPE,

$$
T(z) G^{ \pm}(w) \sim \frac{3 / 2}{(z-w)^{2}} G^{ \pm}(w)+\frac{1}{(z-w)} \partial G^{ \pm}
$$

and

$$
\begin{aligned}
G^{+}(z) G^{-}(w) \sim & \frac{(k+1)(2 k+3)}{(z-w)^{3}}+\frac{3(k+1)}{(z-w)^{2}} H(w) \\
& +\frac{1}{(z-w)}\left[3 H^{2}(w)-(k+3) T(w)+\frac{3(k+1)}{2} \partial H\right] .
\end{aligned}
$$

The OPE between $G^{+}(z)$ and $G^{+}(z)$ (or $G^{-}(z)$ and $G^{-}(z)$ ) does not possess any singular terms. The composite field $H^{2}(z)$ is not conformal, but one may always represent it as the linear combination of a new conformal field with dimension 2 and the stress-energy tensor.

The coefficient $k$ is an arbitrary parameter not equal to -3 . It coincides with the level of the initial $S L(3, \mathscr{R})$ current algebra.

The constructed algebra $W_{3}^{2}$ looks similar to the $N=2$ superconformal algebra [18]. In representation theory one finds different sectors. The twisted sector with half integer modes of $U(1)$-current is related to the twisted $S L(3, \mathscr{R})$ current algebra. The boundary conditions on $G^{ \pm}(z)$ are labeled by one parameter $\eta$. When $z$ goes around the field $|\Phi\rangle$ the bosonic supercurrents get multiplied by phase

$$
G^{ \pm}(z)|\Phi\rangle \rightarrow e^{ \pm 2 \pi i \eta} G^{ \pm}(z)|\Phi\rangle .
$$

These cases $\eta=0$ and $\eta=\frac{1}{2}$ correspond to Neveu-Schwarz and Ramond sectors with half integer modes and integer modes of $G^{ \pm}(z)$ respectively. As in the $N=2$ superconformal case [19] the representations labeled by different $\eta$ are isomorphic to each other due to the $U(1)$ gauge invariance,

$$
\begin{aligned}
G^{+}(z) & \rightarrow e^{-i \alpha(z)} G^{+}(z), \quad G^{-}(z) \rightarrow e^{i \alpha(z)} G^{-}(z), \\
H(z) & \rightarrow H(z)-i \frac{(2 k+3)}{3} \partial \alpha, \\
T(z) & \rightarrow T(z)-i(\partial \alpha) H(z)-\frac{(2 k+3)}{6}(\partial \alpha)^{2} .
\end{aligned}
$$

This gauge invariance is the residual one that survives the reduction.

One can easily deduce the expression for the anomalous dimensions for Neveu- 
Schwarz and Ramond sectors. The representations in question are related to representations of the untwisted $S L(3, \mathscr{R})$ current algebra. For the rational levels $k+3=2 p / q$ there is a finite set of smallest weight states for the $S L(3, \mathscr{R})$ current algebra with weights

$$
\vec{\Lambda}_{r, s}=\sum\left(\left(1-r_{i}\right)(k+3)-\left(1-s_{i}\right)\right) \vec{\omega}_{i}, \quad 1 \leqq s \leqq 2 p-1,1 \leqq r \leqq q,
$$

whose characters transform into each other under modular transformation [20]. Let $\Phi_{\bar{\Lambda}}$ be the smallest weight state from this set. Under reduction it reduces to a primary field with respect to $W_{3}^{2}$ algebra with conformal dimension

$$
\Delta_{\tilde{r}, s}=\frac{1}{4 p q}\left(\sum\left(p \tilde{r}_{i}-q s_{i}\right) \vec{\omega}_{i}\right)^{2}-\frac{(p-q)^{2}}{2 p q}-\frac{1}{16}\left(1-(-11)^{R}\right),
$$

and charge

$$
q_{\tilde{r}, s}=\frac{1}{3}\left(\frac{p}{q}\left(\tilde{r}_{1}-\tilde{r}_{2}\right)-\left(s_{1}-s_{2}\right)\right) \pm \frac{1}{4}\left(1-(-1)^{R}\right)
$$

where $R=0, \tilde{r}=2 r-1$ for the Neveu-Schwarz sector and $R=1, \tilde{r}=2 r$ for the Ramond sector. The negative shift in the vacuum energy in the Ramond sector is due to the bosonic structure of $W_{3}^{2}$ algebra. The values of $\Delta_{\tilde{r}, s}$ and $q_{\tilde{r}, s}$ correspond to highly reducible representations of $W_{3}^{2}$. There is considerable evidence that the fields $\Phi_{r, s}$ with $\tilde{r}$ and $s$ in the region $1 \leqq s \leqq 2 p-1,1 \leqq \tilde{r} \leqq 2 q-1$ make a closed Operator Product Algebra. It seems unlikely that the $W_{3}^{2}$ algebra possesses unitary representations. But if it does they should be in the Kac table of the Virasoro algebra. As the $W_{3}$ algebra the constructed algebra possesses $S L(3, \mathscr{R})$ symmetry which is gauged and hidden. It will be very interesting to understand if there is direct relation between $W_{3}$ and $W_{3}^{2}$ algebras.

\section{Discussion}

The description of extended Virasoro algebras as constrained current algebras seems to be very fruitful. Moreover it provides a classification scheme. One just needs to classify the different type of constraints. The constraints of degree $l$ mentioned in the Introduction are the natural generalizations of the constraints considered in literature. To impose them in a way consistent with the conformal invariance, one has to modify the stress-energy tensor,

$$
T_{\text {improved }}(z)=T_{S L(n)}(z)-\frac{1}{l} \vec{\delta} \partial \vec{H},
$$

where $\vec{\delta}$ is the sum of the fundamental weights of $S L(n, \mathscr{R})$. As with the constrained $S L(3, \mathscr{R})$ current algebra one has to introduce an appropriate number of auxiliary fields with fractional spins $m / l$. For the general case the formulas become very complicated; therefore we restrict ourselves to the case $l=2$. For this case the constraints are

$$
\begin{aligned}
& J_{1,2}^{-}=\chi_{1}, \quad J_{2,3}^{-}=\chi_{2}+\chi_{1}^{+}, \quad \cdots \quad J_{n-1, n}^{-}=\chi_{n-2}^{+}, \\
& J_{1,3}^{-}=J_{2,4}^{-}=\cdots=J_{n-2, n}^{-}=1, \quad J_{i, j}^{-}=0 \quad j \geqq i+2,
\end{aligned}
$$

where $\left(\chi_{i}, \chi_{i}^{+}\right)$are auxiliary fields of dimension $\left(\frac{1}{2}, \frac{1}{2}\right)$. 
The discussion follows precisely the $S L(3, \mathscr{R})$ case. The constrained WZW-model is gauge invariant under an $n(n-1) / 2$ parameter group. Therefore one should expect that the reduced theory possesses $3 n-5$ degrees of freedom. Again choosing appropriate gauge fixing conditions one can eliminate the auxiliary fields $\left(\chi_{i}, \chi_{i}^{+}\right)$. The geometric action coincides with the WZW action evaluated under the constraints. Its right sector is invariant with respect to chiral algebra. In principle it is possible to work out the classical symmetry of the reduced theory in each particular case. The classical bosonization rules for the $W_{n}$ algebra are given by the Miura [5] transformation which is nothing else but the relation between two different choices of gauge slice. It would be very nice to have some analog of the Miura transformation for the general case.

It is easy to evaluate the conformal anomaly for the reduced theory,

$$
c_{\text {reduced }}=\frac{\left(n^{2}-1\right) k}{(k+n)}-\frac{1}{4}\left(n^{3}-n\right) k-(n-2)+c_{\text {ghost }} \text {. }
$$

The ghost contribution $c_{\text {ghost }}$ can be easily computed. Substituting it into (50) and parameterizing the level $k$ as $k+n=2 p / q$ we obtain

$$
c_{\text {reduced }}=1-\frac{1}{2}\left(n^{3}-n\right) \frac{(p-q)^{2}}{p q} .
$$

Surprisingly all values are less than one.

The total Hilbert space is the product of $S L(n, \mathscr{R})$ representation space, the Fock space of the auxiliary fields and the ghost Fock space. Again there is a nilpotent BRST operator which can be made linear in constraints by conjugation. To obtain the reduced Hilbert space we impose the constraints using the BRST formalism. Following the arguments of the previous section one can immediately guess the reduced screening operators ${ }^{1}$,

$$
\begin{aligned}
V_{1}^{ \pm} & =\left(\phi_{1}(z)^{n_{ \pm}} \exp \left(\alpha_{ \pm} \vec{e}_{1} \cdot \vec{\varphi}(z)\right),\right. \\
V_{2}^{ \pm} & =\left(\phi_{2}(z)+\phi_{1}^{+}(z)\right)^{n_{ \pm}} \exp \left(\alpha_{ \pm} \vec{e}_{2} \cdot \vec{\varphi}(z)\right), \\
& \vdots \\
V_{n-2}^{ \pm} & =\left(\phi_{n-2}(z)+\phi_{n-3}^{+}(z)\right)^{n_{ \pm}} \exp \left(\alpha_{ \pm} \vec{e}_{n-2} \cdot \vec{\varphi}(z)\right), \\
V_{n-1}^{ \pm} & =\left(\phi_{n-2}^{+}(z)\right)^{n_{ \pm}} \exp \left(\alpha_{ \pm} \vec{e}_{n-1} \cdot \vec{\varphi}(z)\right),
\end{aligned}
$$

when $n_{-}=1, n_{+}=-(k+n), k$ is the level of the initial current algebra. The $(n-1)$ dimensional scalar field $\vec{\varphi}$ bosonizes the Cartan subalgebra of the $S L(n, \mathscr{R})$. The bosonic fields $\left(\phi_{i}, \phi_{i}^{+}\right)$are expressed as some particular linear combinations of $\left(\beta_{j}, \gamma_{j}\right)$ fields that bosonize the Borel subalgebra of $S L(n, \mathscr{R})$. Again one can construct the chiral algebra as the commutant of the screening operators. One can easily check that the $U(1)$ current and the stress-energy tensor are

$$
\begin{aligned}
& H(z)=\alpha_{+}\left(\vec{\omega}_{n-1}-\vec{\omega}_{1}\right) \partial \vec{\varphi}+\sum_{i} \phi_{i} \phi_{i}^{+}, \\
& T(z)=T_{\text {free }}(z)+\alpha_{0} \vec{\delta} \partial^{2} \vec{\varphi},
\end{aligned}
$$

\footnotetext{
${ }^{1}$ Compare with screening operators for $W_{n}[4]$
} 
where $\alpha_{+}=\sqrt{2 k+2 n}, \alpha_{0}=(k+n-1) / \alpha_{+}$. The bosonization rules for other operators are quite complicated. One can verify that the conformal anomaly for this stress-energy tensor is given by Eq. (51). It would be very interesting to construct these algebras explicitly.

Acknowledgements. The author would like to thank I. Bars, J. Cohn, E. Frenkel, D. Gross, A. Polyakov, A. Shapere, R. Silvotti, E. Verlinde and A. Zamolodchikov for comments and discussions. This work was supported by the Alan T. Warterman Award under grant number PHY-881752188.

\section{References}

1. Belavin, A.: On the Connection between Zamolodchikov's $W$-algebras and Kac-Moody Algebras. In: Kawamoto, N., Kugo, T. (eds.) Quantum String Theory, Proceedings of the Second Yukawa Memorial Symposium, Nishinomiya, Japan 1987, Proceedings in Physics vol. 31, pp. 132-137, Berlin, Heidelberg, New York: Springer 1989; KdV-type equations and W-Algebras, handwritten manuscript, 1988

2. Bershadsky, M., Ooguri, H.: Hidden $S L(n)$ symmetry in the conformal field theories. Commun. Math. Phys. 126, 49-83 (1989)

3. Zamolodchikov, A.: Infinite additional symmetries in two-dimensional quantum field theories. Theor. Math. Phys. 63, 1205-1203 (1985)

4. Fateev, V., Lykyanov, S.: The models of two-dimensional conformal quantum field theory with $Z_{n}$-symmetry. Int. J. Mod. Phys. A3, 507-520 (1988)

5. Drinfeld, V., Sokolov, V.: Lie algebras and equations of Korteweg-de Vries type. J. Sov. Math. 30, 1975-2036 (1984)

6. Polyakov, A.: Gauge transformations and diffeomorphisms. Int. J. Mod. Phys. A5, 833 (1990)

7. Douglas, M.: Strings in less than one dimension and generalized KdV hierarchies. Rutgers Preprint RU-89-51, 1989

8. Moore, G.: Geometry of the string equations. Yale Preprint YCTP-P4-90, 1990

9. Bershadsky, M., Ooguri, H.: Hidden $\operatorname{OSp}(n, 2)$ symmetries in superconformal field theories. Phys. Lett. 229B, 374-378 (1989)

10. Alekseev, A., Shatashivili, S.: Path integral quantization of the coadjoint orbits of the Virasoro group and 2-D gravity. Nucl. Phys. B323, 719 (1989)

11. Bernard, D., Felder, G.: Fock representations and BRST cohomology in $S L(2)$ current algebra. Commun. Math. Phys. 127, 145 (1990)

12. Feigin, B., Frenkel, E.: Representations of affine Kac-Moody algebras, Bosonization and resolutions. Lett. Math. Phys. 19, 307-319 (1990)

13. Feigin, B., Frenkel, E.: Representations of affine Kac-Moody algebras and Bosonization. Moscow Preprint, 1989

14. Bouwknegt, P., McCarthy, J., Pilch, K.: Free field realization of WZW-models; BRST complex and its quantum group structure. Phys. Lett. 324B, 297-303 (1990)

15. Bouwknegt, P., McCarthy, J., Pilch, K.: Quantum group structure in the Fock space resolutions of $S L(n)$ representations. Preprint MIT-CTP-1797, 1989

16. Felder, G.: BRST Approach to minimal models. Nucl. Phys. B317, 215 (1989)

17. Kugo, T., Ojima, I.: Manifestly covariant canonical formalism of the Yang-Mills field theories I. Prog. Theor. Phys. 60, 1869 (1978)

18. Ademolo, M. et al.: Supersymmetric strings and color confinement. Phys. Lett. 62B, 105 (1976)

19. Schwimmer, A., Seiberg, N.: Comment on the $N=2,3,4$ superconformal algebras in two dimensions. Phys. Lett 184B, 191-196 (1987)

20. Kac, V., Wakimoto, M.: Modular invariant representations of infinite dimensional Lie algebras and superalgebras. Preprint MIT, 1988 